\title{
Actinomycosis and Sialolithiasis in Submandibular Gland
}

\author{
Jin Seok Kang ${ }^{1}$, \\ Hwan Jun $\mathrm{Choi}^{2}$, \\ Min Sung Tak ${ }^{1}$ \\ ${ }^{1}$ Department of Plastic and \\ Reconstructive Surgery, Soonchunhyang \\ University Seoul Hospital, Seoul; \\ ${ }^{2}$ Department of Plastic and \\ Reconstructive Surgery, Soonchunhyang \\ University Cheonan Hospital, \\ Soonchunhyang University College of \\ Medicine, Cheonan, Korea
}

No potential conflict of interest relevant to this article was reported.

\begin{abstract}
Actinomycosis is a subacute or chronic suppurative infection caused by Actinomyces species, which are anaerobic Gram-positive bacteria that normally colonize the human mouth and digestive and urogenital tracts. Cervicofacial actinomycosis is the most frequent clinical form of actinomycosis, and is associated with odontogenic infection. Characterized by an abscess and mandibular involvement with or without fistula, but the cervicofacial form of actinomycosis is often misdiagnosed because the presentation is not specific and because it can mimic numerous infectious and non-infectious diseases, including malignant tumors. We report a rare case of actinomycosis infection with coexisting submandibular sialolithiasis. The patient presented with a $1 \times 1 \mathrm{~cm}$ abscess-like lesion below the lower lip. Punch biopsy of the lesion revealed atypical squamous cell proliferation with infiltrative growth, suggestive of squamous cell carcinoma. The patient underwent wide excision of this lesion, where the lesion was found to be an abscess formation with multiple submandibular sialolithiases. The surgical specimen was found to contain Actinomyces without any evidence of a malignant process. We assumed that associated predisposing factors such as poor oral hygiene may have caused a dehydrated condition of the oral cavity, leading to coexistence of actinomycosis and sialolithiasis.
\end{abstract}

Keywords: Actinomycosis / Sialolithiasis

\section{INTRODUCTION}

Actinomycosis is a subacute or chronic suppurative infection caused by Actinomyces species, which are anaerobic Gram-positive bacteria that normally colonize the human mouth and digestive and urogenital tracts [1]. To date, multiple clinical features of actinomycosis have been described, and the clinical types of actinomycosis are cervicofacial, thoracic, abdominal, and female genitalia. Cervicofacial actinomycosis is the most common form and comprises about $50 \%$ of all reported cases [2]. The most frequently affected cervicofacial sites are the parotids and the mandible [3]. Cervicofacial actinomycosis is associated with an abscess

\footnotetext{
Correspondence: Hwan Jun Choi

Department of Plastic and Reconstructive Surgery, Soonchunhyang University Cheonan Hospital, Soonchunhyang University College of Medicine,

31 Suncheonhyang 6-gil, Dongnam-gu, Cheonan 330-721, Korea

E-mail: medi619@hanmail.net

*This work was supported by Soonchunhyang University Research Fund.

Received January 16, 2015 / Revised February 27, 2015 / Accepted April 2, 2015
}

and mandibular involvement with or without fistula. Often misdiagnosed, clinical presentation is not specific, and the lesions can mimic numerous infectious and non-infectious diseases, including malignant tumors [1]. We report a case of actinomycosis of the lower lip with multiple submandibular gland calcifications that mimicked squamous cell carcinoma.

\section{CASE REPORT}

An 87-year-old woman presented to the outpatient dermatology clinic with a complaint of a progressive abscess-like lesion on the lower lip which was painful and discharging pus (Fig. 1). The lesion had developed about 1 year ago and initially it was non-tender but later it became tender. Physical examination revealed an abscess-like lesion measuring about $1 \times 1 \mathrm{~cm}$ below the lower lip and multiple palpable hard masses on the lower lip. Purulent yellow exudate was discharged upon expression. No cervical lymphadenopathy was noted. There was no history of trauma, but the 
patient had poor oral hygiene with a relatively dehydrated cavity. Punch biopsy of the lesion revealed atypical squamous cell proliferation with infiltrative growth, suggestive of squamous cell carcinoma. Furthermore, cervical computed tomography identified multiple microcalcifications in the minor salivary gland and the submandibulargland (Fig. 2).

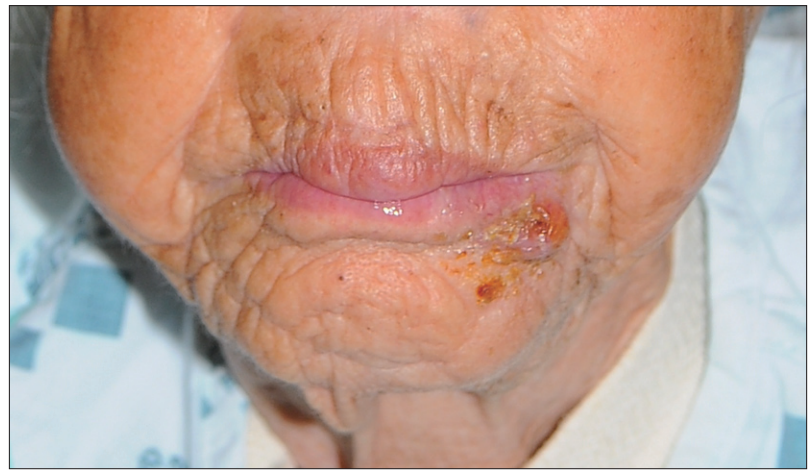

Fig. 1. Abscess-like lesion measuring about $1 \times 1 \mathrm{~cm}$ below the lower lip.
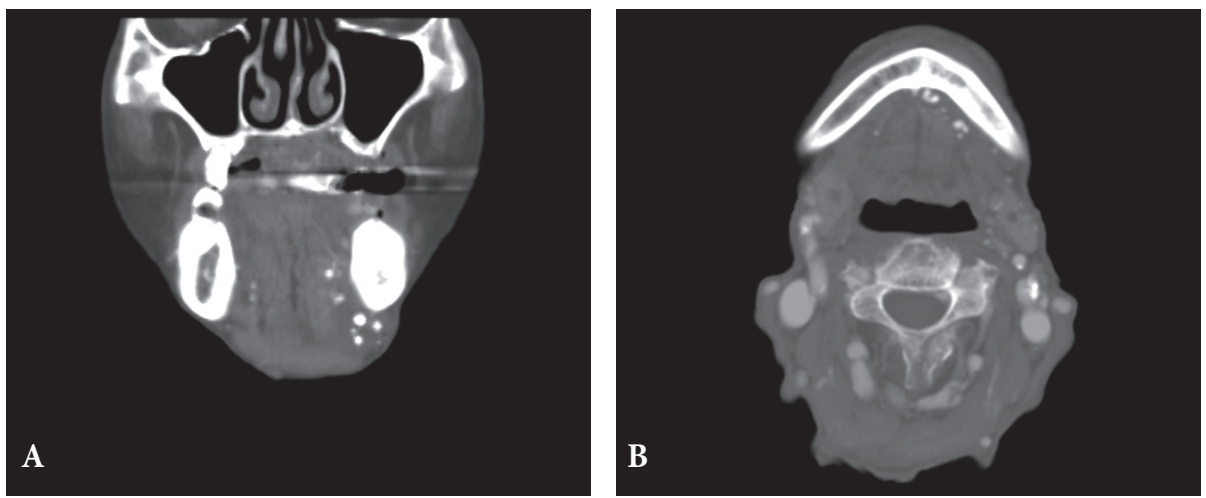

Fig. 2. Multiple submandibular gland sialolithiasis on computed tomography. (A) Coronal view, (B) axial view.

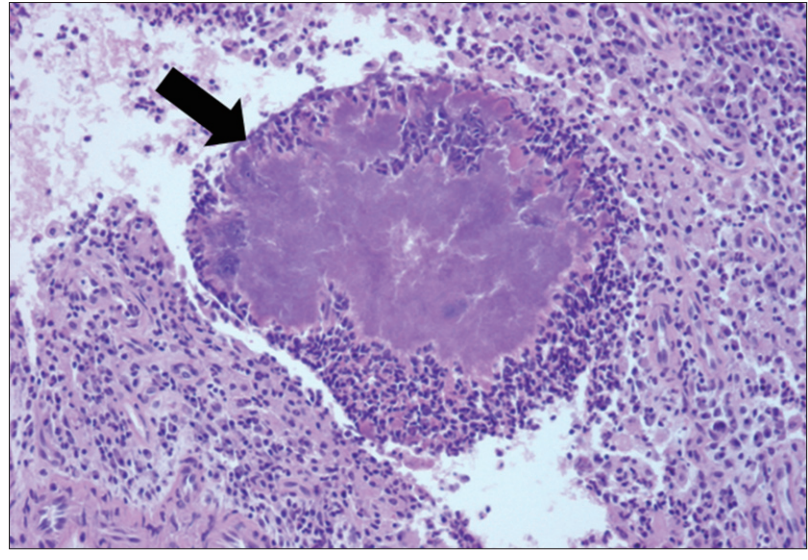

Fig. 3. Histopathologic image. Suppurative inflammation including a bacterial colony (arrow) with abscess formation $(\mathrm{H} \& \mathrm{E}, \times 200)$.
The lesion was widely excised for tentative diagnosis of squamous carcinoma. The lesion was resected with a 5-mm margin, and a separate $0.2 \times 0.3 \mathrm{~cm}$ mass was removed from the lower lip. The resulting defect was reconstructed using a local advancement flap. On pathologic examination, suppurative inflammation including a bacterial colony with thread-like morphology was observed. This was confirmed to be actinomycosis abscess, and there was no evidence of a malignant process on final review (Figs. 3, 4). Postoperatively, high-dose amoxicillin (6 g/day orally) was prescribed for 2 weeks. There were no notable complications such as disruption, recurrence, and deformity. At 2 months follow-up visit, the wound had completely healed (Fig. 5).

\section{DISCUSSION}

Cervicofacial actinomycosis is the most frequent clinical form of

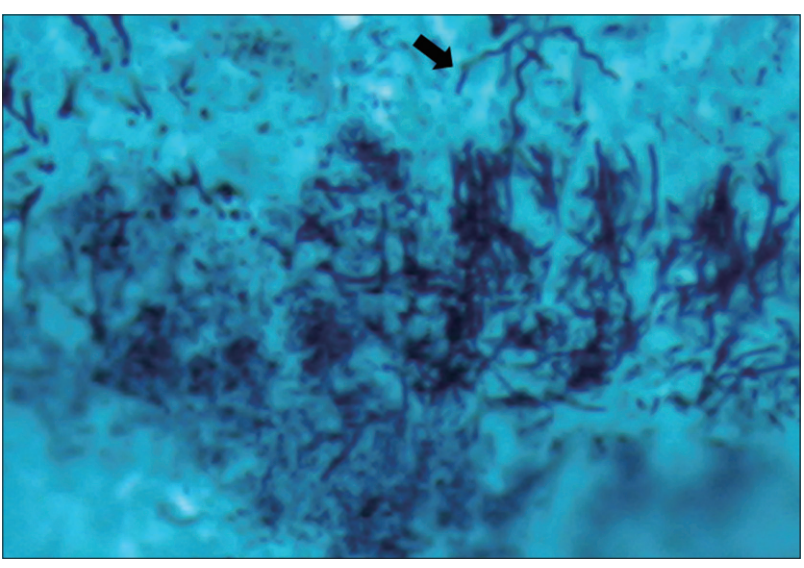

Fig. 4. Histopathologic image. Thread-like morphology (arrow) of actinomycosis (Gomori's methenamine silver stain, $\times 400$ ). 


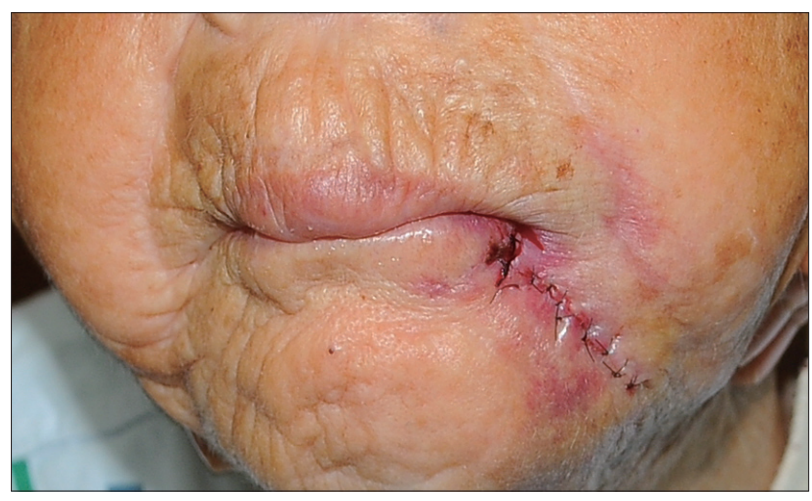

Fig. 5. Postoperative views at 2 days after operation.

actinomycosis, and is associated with odontogenic infection. Predisposing conditions include poor oral hygiene, oral mucosal trauma, and dental intervention [1]. The typical presentation is formation of an abscess with sinus tracts on the skin surface or the oral mucosa and hardening of the soft tissue. Also, this form of actinomycosis involves tissues surrounding the upper or lower mandible, including the mandible itself $(50 \%)$, cheeks $(15 \%)$, chin (15\%), and submaxillary ramus and angle (10\%) [1]. Computed tomography and magnetic resonance imaging (MRI) is helpful in elucidating any osteomyelitis and osteolysis with extensive inflammatory changes in the surrounding soft tissue extending to the skin, but they may not provide an accurate diagnosis for the cause of infection [1]. Actinomycosis infections are challenging to diagnose, and is often misdiagnosed because it can mimic numerous infectious and non-infectious diseases, including nocardiosis or mycobacterial infection or malignant tumors [1]. The gold standard for diagnosing cervicofacial actinomycosis is histological examination and bacterial culture, but the surgeon should clinically suspect cervicofacial actinomycosis based on typical signs such as a progressive painless indurated mass, evolving into multiple abscesses with draining sinus tracts on the skin surface or oral mucosa and mandibular osteomyelitis [1].

For cervicofacial actinomycosis, surgical management can be required for drainage of abscess, marsupialization of chronic sinus tracts, excision of fibrotic lesion, and debridement of necrotic bone tissue [1]. Most actinomyces species are susceptible to betalactams, and the treatment of choice is a prolonged course of oral amoxicillin [1]. Acceptable alternatives include clindamycin, mac- rolides, and doxycycline, which has a better bone penetration. The long treatment course can likely be shortened if the infected tissues were surgically removed in the absence of bone involvement [1]. Calcific lesions in the major salivary glands are related to sialolithiasis and are found in chronic inflammatory conditions. They are usually discovered during routine dental or head and neck radiographic examinations, but the differential diagnosis should include a wide spectrum of diseases such as calcified lymph nodes, tuberculosis infection, calcified vascular lesions presenting as vascular malformations, and finally, metastasis from distinct calcifying neoplasms [4]. Actinomycosis of the submandibular gland is rare, and it causes sialadenitis and sialolithiasis that mimic a malignant tumor [5]. In our case, we could not identify any correlation between multiple submandibular gland sialolithiasis and the squamous cell carcinoma biopsy result before the final pathological result. However, the presence of actinomycosis infection from operative specimen may have caused a chronic inflammatory condition of the salivary gland, which finally led to multiple sialolithiasis of submandibular gland. With regard to actinomycosis infection, poor oral hygiene may have been a significant cause. The dehydrated condition of oral cavity may have caused formation of salivary duct stones. Under this condition, the saliva becomes more concentrated and also leads to mucosal breakdown, which is a predisposing condition for actinomycosis infection. Also, chronic inflammation due to infection with tuberculosis or actinomycosis may have led to sialolithiasis. Uslu et al. [6] reported a case that actinomycosis of the submandibular gland associated with sialolithiasis. The patient had a $4 \times 3 \mathrm{~cm}$ mass in the submandibular region, which was initially treated with penicillin for nine months. However, the antibiotic treatment did not improve clinical course, and the mass did not decrease in size. As such, submandibular gland excision was performed [6]. In our case, we performed wide excision and postoperatively prescribed highdose amoxicillin (6 g/day orally) for 2 weeks. In consideration of patient's age and small multiple sialolithiasis, we did not perform submandibular gland excision. In conclusion, clinical presentation is rare for actinomycosis and sialolithiasis involving the submandibulargland and the minor salivary gland. 


\section{REFERENCES}

1. Valour F, Senechal A, Dupieux C, Karsenty J, Lustig S, Breton P, Gleizal A, Boussel L, Laurent F, Braun E, Chidiac C, Ader F, Ferry T. Actinomycosis: etiology, clinical features, diagnosis, treatment, and management. Infect Drug Resist 2014;7:183-97.

2. Kolm I, Aceto L, Hombach M, Kamarshev J, Hafner J, Urosevic-Maiwald M. Cervicofacial actinomycosis: a long forgotten infectious complication of immunosuppression-report of a case and review of the literature. Dermatol Online J 2014;20:22640.

3. Palonta F, Preti G, Vione N, Cavalot AL. Actinomycosis of the masseter muscle: report of a case and review of the literature. J Craniofac Surg 2003;14:915-8.

4. Bar T, Zagury A, London D, Shacham R, Nahlieli O. Calcifications simulating sialolithiasis of the major salivary glands. Dentomaxillofac Radiol 2007;36:59-62.

5. Bialek EJ, Jakubowski W, Zajkowski P, Szopinski KT, Osmolski A. US of the major salivary glands: anatomy and spatial relationships, pathologic conditions, and pitfalls. Radiographics 2006;26:745-63.

6. Uslu C, Oysu C, Ulkumen B. Coexistence of actinomycosis and sialolithiasis in the submandibular gland. Kulak Burun Bogaz Ihtis Derg 2008;18:257-9. 